\title{
Cross dipoles rectenna for microwave applications
}

\author{
A. Okba ${ }^{1}$, S. Charlot ${ }^{1}$, P-F Calmon ${ }^{1}$, A. Takacs ${ }^{1,2}$, H. Aubert ${ }^{1,3}$
}

${ }^{1}$ CNRS, LAAS, 7 avenue du colonel Roche, F-31400, Toulouse, France

${ }^{2}$ Univ de Toulouse, UPS, LAAS, F-31400, Toulouse, France

${ }^{3}$ Univ de Toulouse, INP, LAAS, F-31400, Toulouse, France

\begin{abstract}
This paper addresses recent results obtained in the $\mathrm{Ku}$ band by using an innovative cross dipoles rectenna topology. The targeted application is the harvesting of the spill-over losses of microwave antennas for powering autonomous wireless sensors used for satellite health monitoring. The experimental results demonstrate that this compact antenna can provide at least $1 \mathrm{~mW}$ of DC power when it is illuminated by an incident electric field of $38 \mathrm{~V} / \mathrm{m}$ or higher.
\end{abstract}

Keywords - electromagnetic energy harvesting, wireless power transfer, rectennas, printed dipoles array, satellite application.

\section{INTRODUCTION}

Recently, the electromagnetic harvesting of the spill-over losses of microwave antennas was identified as a realistic solution to power autonomous wireless sensors for health monitoring of the antenna panels of the broadcasting satellites [1]. This paper presents recent results in $\mathrm{Ku}$ band obtained from an innovative rectenna topology based on four printed cross dipoles antenna array and uses a low cost Schottky diode [2]. Rectenna using a cross dipole antenna was also reported in [1],[3] while a rectenna using a two cross dipoles array was reported in [4]. The rectenna topology and the design methodology are presented in Section II while Section III shows the experimental results obtained by using the manufactured rectenna.

\section{RECTENNA TOPOLOGY AND DESIGN}

The proposed topology is composed of: four cross dipoles array (4CDAA) and a silicon Schottky diode - Skyworks SMS7630 [2]. The PCB layout and a photo of the manufactured rectenna are shown in Fig. 1.The antenna array and the diode are implemented on the top of the PCB while the RF shunt capacitor is mounted on the bottom side of PCB. A reflector metallic plate is added on the bottom side in order to improve the antenna gain. This compact topology (its size is fixed by the size of the antenna array) is an extension of a rectenna using two cross dipoles antenna array 2CDAA reporteded in [4] and a non-resonant matching technique was used. This non-resonant matching is implemented by properly controlling (i) the input impedance of the antenna array and (ii) the input impedance of the rectifier itself. The rectifier consists of the diode and the low pass RC filter (composed of the shorting capacitor and the load itself). The 4CDAA can be viewed as an array formed by four cross dipoles printed on the PCB. Each cross dipole consists of two dipoles in cross configuration as shown in Fig. 1. The substrate used is Rogers
6002 (substrate thickness: $0.508 \mathrm{~mm}$, relative dielectric permittivity: 2.94, dielectric loss tangent: 0.0012).
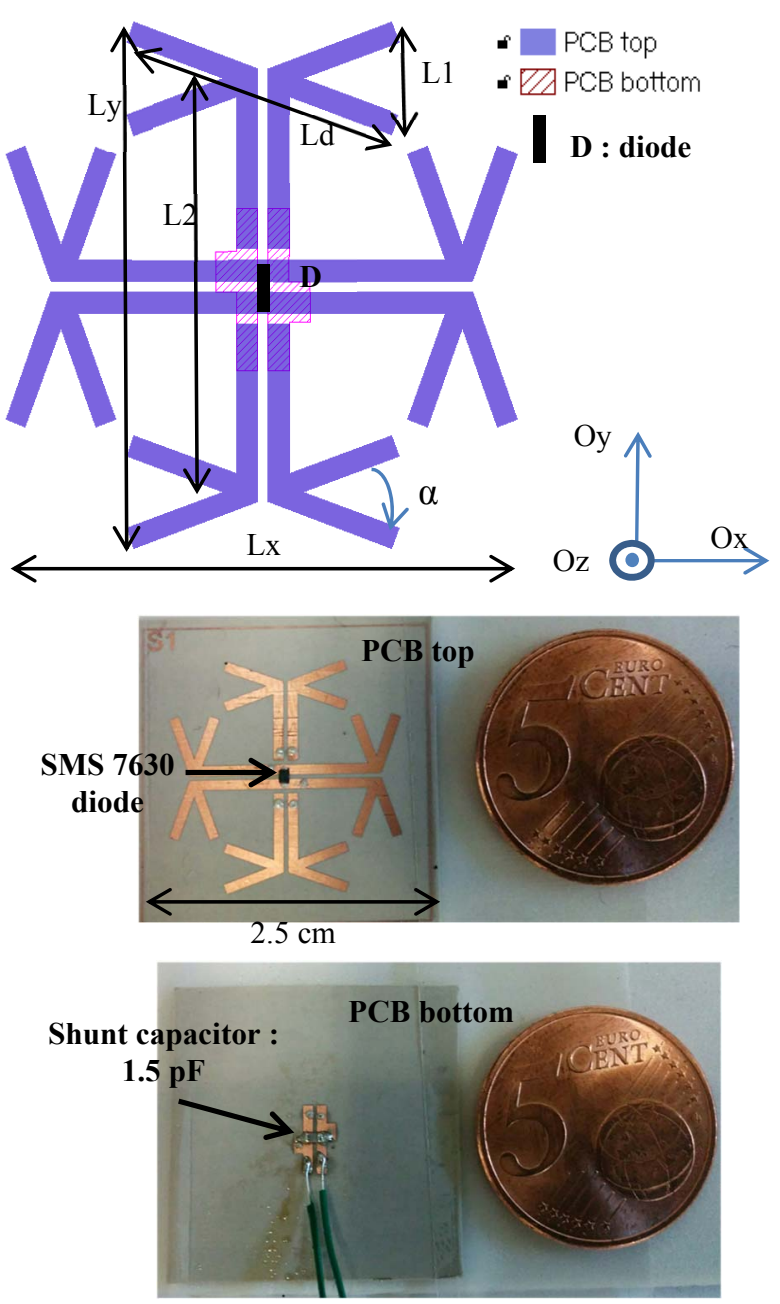

Fig. 1. Top view (not to scale) of the layout of the 4CDAA rectenna, its main geometrical dimensions and the photos of top and bottom side of the manufactured rectenna. The wires shown here was used to connect a variable resistance in order to reach the optimal load.

The maximum theoretical gain (Gmax in $\mathrm{dBi}$ ) of such an array antenna can be computed using the following expresion:

$$
G \max =10 \cdot \log (8)+G d=11.18 d B i(1)
$$


where $\mathrm{Gd}=2.15 \mathrm{dBi}$ is the gain of a lossless halfwavelength dipole. This gain can be increased by $3 \mathrm{~dB}$ when a metallic reflector is properly positioned below the antenna surface. Thus the expected maximum gain is $14.18 \mathrm{dBi}$. This theoretical gain should be considered as an upper bound for any practical 4CDAA design. There are no accurate analytical formulas to design such an array but some rules can be used in order to start the design. The length of each dipole (Ld in Fig 1) should be approximately half wavelength and the distance between the center of two opposite cross dipoles (L2 in Fig.1) should be an integer multiple of half wavelength. The angle between the dipoles ( $\alpha$ in Fig 1) impacts the wave polarization and the maximum achievable gain. Linear or circular polarization can be obtained by using this topology as function of the relative phase of waves exciting the crossed dipoles. Coplanar strip lines are used to feed each cross dipole. We target here a linear polarization. Thus the diode should be mounted in the center of the array as represented in Fig. 1.
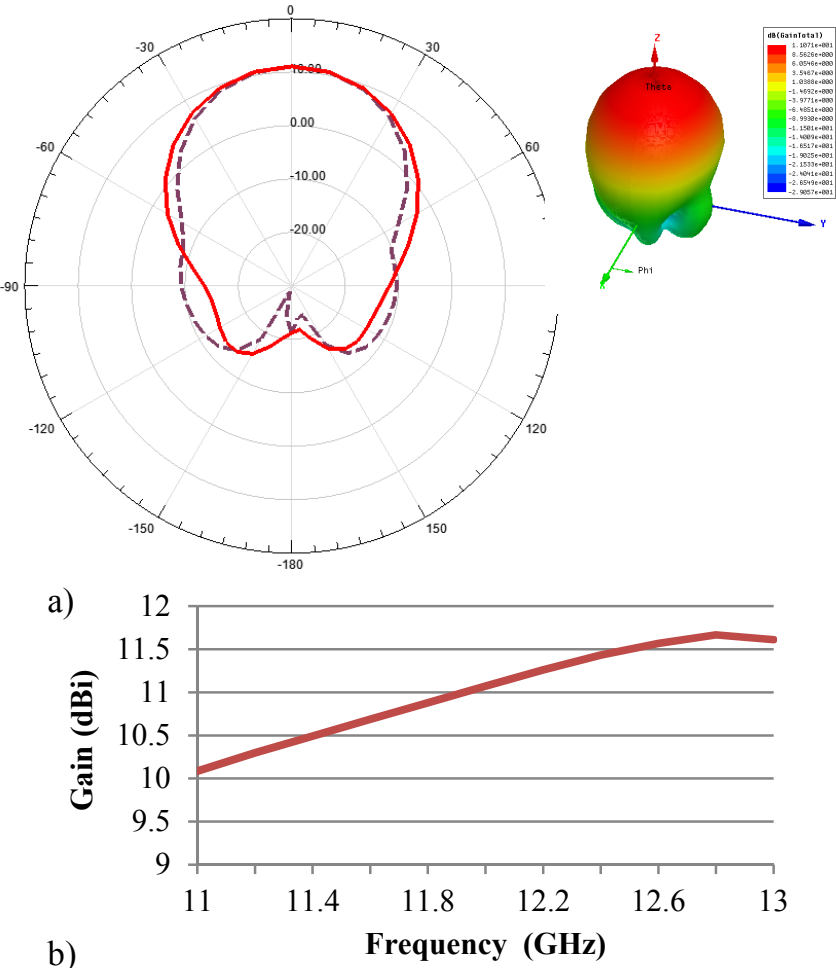

b)

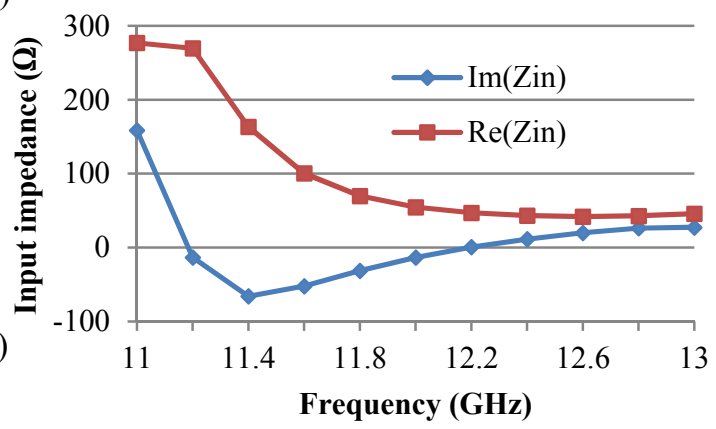

Fig. 2. a) Simulated (HFSS) gain in the $x O z$ plane $\left(p h i=0^{\circ}\right)$ - continous line - and $\mathrm{xOy}$ plane $\left(\mathrm{phi}=90^{\circ}\right)$ - dashed-line of the 4CDAA at the frequency of $12 \mathrm{GHz}$, the inset shows the 3D radiation pattern b) Simulated (HFSS) maximum gain of the 4CDAA vs the frequency, c) the input impedance presented by the 4CDAA on the input port of the diode vs frequency.
The distance between the mounting position of the diode and each dipole should be in the range of half wavelength. Six metallic via holes were used to connect the top and the bottom side of the PCB. A shunt capacitor is mounted on the bottom side of the PCB. The main dimensions of the fabricated (optimized) rectenna are: $\mathrm{L} 1=4 \mathrm{~mm}, \mathrm{~L} 2=15.3 \mathrm{~mm}, \mathrm{~L} 3=10.6$ $\mathrm{mm}, \mathrm{Lx}=\mathrm{Ly}=19.5 \mathrm{~mm}$. Intensive full-wave electromagnetic simulations were performed by using Ansys HFSS software in order to optimize the 4CDAA. The optimized 4CDAA exhibits a maximum gain (over $\mathrm{Oz}$ axis) of $11 \mathrm{dBi}$ as represented in Fig. 2 a) and Fig 2 b). The simulated input impedance is shown in the Fig. 2 c).

A generic electrical diode model existing in the ADS software was customized with the following parameters [5][6]: ohmic resistance $R_{s}=20 \Omega$, junction capacitance $C_{j 0}=$ $0.14 \mathrm{pF}$, saturation current $\mathrm{I}_{\mathrm{s}}=5 \mu \mathrm{A}$, forward voltage $\mathrm{V}_{\mathrm{f}}=$ $0.34 \mathrm{~V}$, reverse breakdown voltage $\mathrm{B}_{\mathrm{v}}=2 \mathrm{~V}$ and the current at reverse breakdown voltage $\mathrm{I}_{\mathrm{bv}}=0.1 \mathrm{~mA}$. The ADS simulation model is represented in Fig. 3. The PCB located on the bottom side of the PCB was simulated by using standard printed PCB lines and via holes founded in the ADS library. The harmonic balance port was charged by the input impedance (simulated in HFSS and depicted in Fig. 2 c) presented by 4CDAA at the input port of the diode.

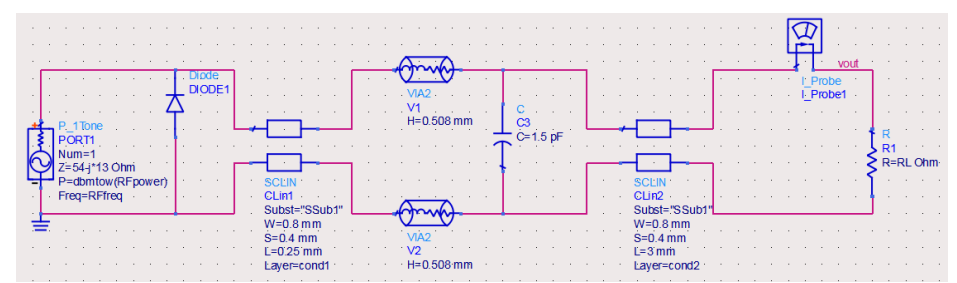

Fig. 3. ADS circuital simulation model.

The initial simulations performed by using this model demonstrated that the maximum DC power was obtained at the frequency of $12 \mathrm{GHz}$ approximately. Nevertheless the ADS simulations shows convergence issues of the harmonic balance engine and an important sensitivity of the DC power delivered to the load (RL) as function of the length of the printed PCB line. Thus we decided to manufacture a prototype and to keep as compact as possible the PCB layout of the bottom side in order to avoid any uncontrolled coupling between the 4CDAA (bottom side) and the PCB layout of the bottom side.

\section{EXPERIMENTAL RESULTS AND DISCUSSION}

The experimental setup shown in the Fig. 4 was used to characterize the rectenna. A microwave signal generated from an Anritsu MG3694B generator was injected at the input of a horn antenna which illuminated the rectenna under test with a linearly polarized E-field. An automatic acquisition routine was implemented in Labview software from National Instruments to speed-up the acquisition process. The harvested DC voltage was measured by using a DC multimeter. The DC power can be computed from the measured DC voltage as long as the load is known. The measured loss due to the coaxial cable and connectors between antenna and the signal 
generator was in the range of $3 \mathrm{~dB}$ in the operating frequency band.

The efficiency $\eta$ (in \%) of the rectenna can be computed by using the following definition [7]:

$$
\eta=\frac{P_{D C}}{S \cdot A_{e f f}} \cdot 100=\frac{4 \cdot \pi \cdot P_{D C}}{S \cdot G_{R} \cdot \lambda^{2}} \cdot 100
$$

where $\mathrm{P}_{\mathrm{DC}}$ is the harvested $\mathrm{DC}$ power, $\mathrm{S}$ is the incident electromagnetic power density $\mathrm{A}_{\mathrm{eff}}$ is the antenna effective area, $G_{R}$ is the gain of the (rectenna's) antenna and $\lambda$ is the wavelength of the illuminating electromagnetic wave.

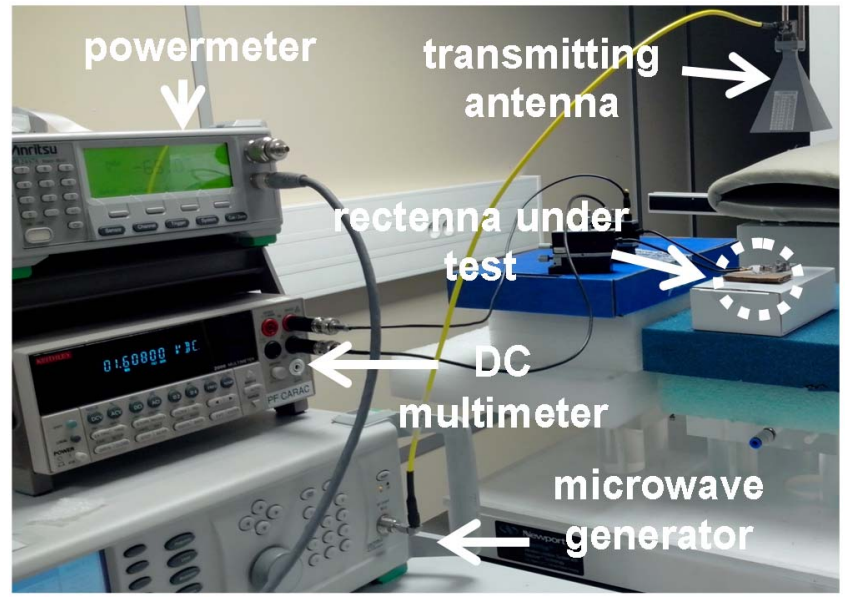

Fig. 4. Experimental setup used to characterize the rectenna

The power density $\left(\mu \mathrm{W} / \mathrm{cm}^{2}\right)$ can be computed as a function of E-field effective value $\mathrm{E}(\mathrm{V} / \mathrm{m})$ on the antenna surface or as a function of the RF power $P_{t}$ injected to the input of the transmitting horn antenna of gain $\mathrm{G}_{\mathrm{t}}$ and positioned at the distance $d$ from the rectenna, as follows:

$$
S=\frac{E^{2}}{120 \cdot \pi} \cdot 100=\frac{30 \cdot P_{t} \cdot G_{t}}{d^{2} \cdot 120 \cdot \pi} \cdot 100
$$

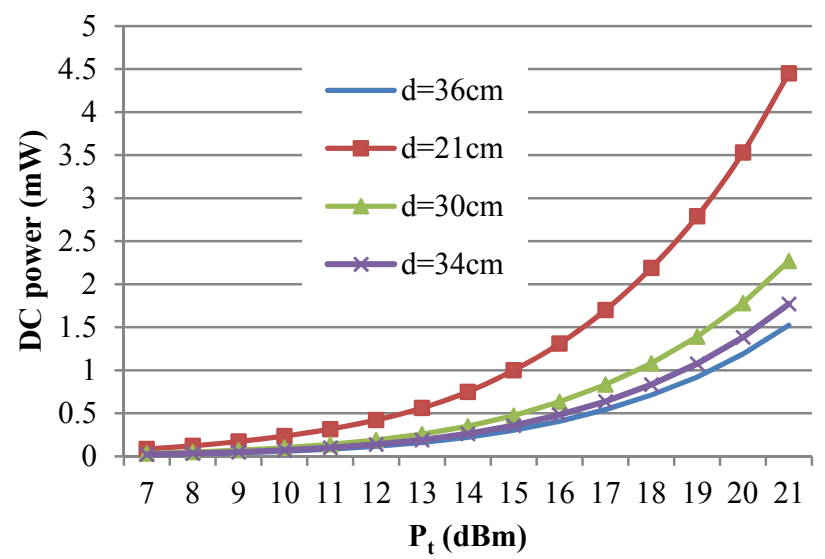

Fig. 5. Measured DC power at the input port of a resistive load $(R L=300 \Omega)$ as function of the RF power injected into the transmiting horn antenna.

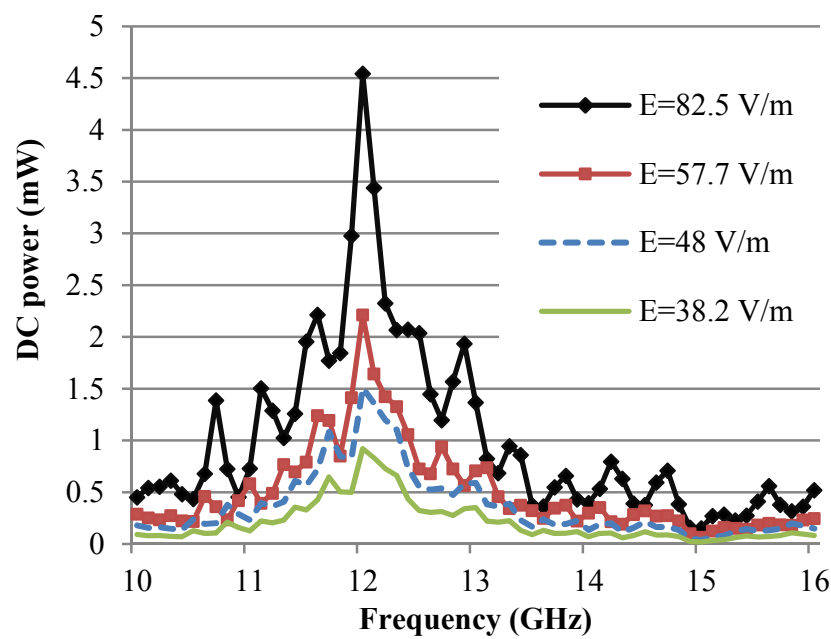

Fig. 6. Measured DC power at the input port of a resistive load $(R L=300 \Omega)$ as function of frequency for several value of the E-field

Fig. 5 displays the measured DC power on a load of $\mathrm{RL}=300 \Omega$ as function of the RF power injected in the transmitting horn antenna (of gain $\mathrm{G}_{\mathrm{R}}=19 \mathrm{dBi}$ ) for several distances between the transmitting horn antenna and the rectenna under test. We note that power up to $4.5 \mathrm{~mW}$ can be harvested by using the proposed 4CDAA rectenna.

The E-field and the power density illuminating the rectenna can be computed as function of the $\mathrm{G}_{\mathrm{t}}$ and $\mathrm{d}$ by using eq. (3). Fig. 6 shows the measured harvested DC power at the input port of a resitive load $(\mathrm{RL}=300 \Omega)$ as function of the frequency for several values of the E-field. The maximum DC power was measured at $12 \mathrm{GHz}$ as represented in Fig. 6.

The optimal load was also determined. The electric field illuminating the rectenna under test was fixed at $\mathrm{E}=48 \mathrm{~V} / \mathrm{m}$ by using the following configuration: $\mathrm{d}=36 \mathrm{~cm}, \mathrm{G}_{\mathrm{t}}=19 \mathrm{dBi}$, power delivered by the microwave generator: $24 \mathrm{dBm}$, injected power at the input of the transmitting horn antenna: $21 \mathrm{dBm}$. The obtained results are depicted in Fig. 7. We remark that the maximum DC power is obtained for a load impedance $\mathrm{RL}=300 \Omega$.

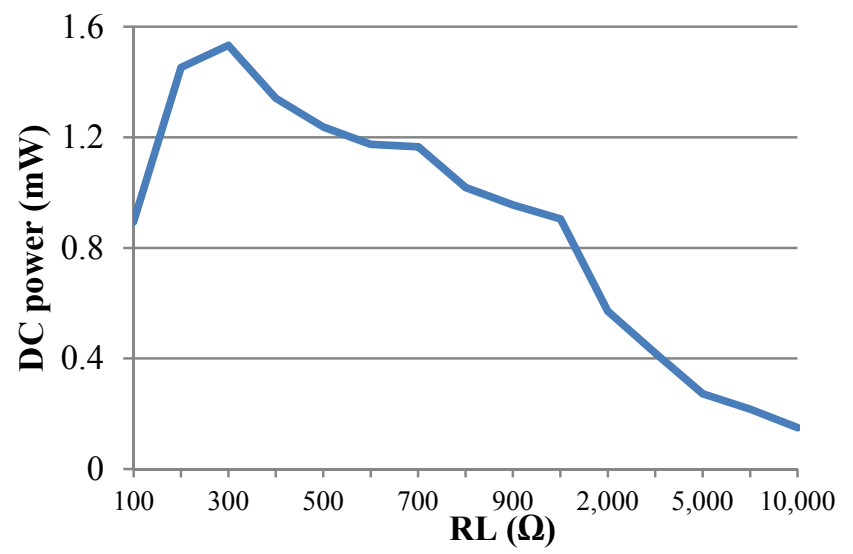

Fig. 7. Measured DC power as function of the load impedance $(E=48 \mathrm{~V} / \mathrm{m}$, $\mathrm{f}=12 \mathrm{GHz}$ ) 
The harvested DC power for $\mathrm{RL}=300 \Omega$ as function of $\mathrm{E}$ field illuminating the 4CDAA at the frequency of $12 \mathrm{GHz}$ is represented in Fig. 8. From a practical point a view at least 1 $\mathrm{mW}$ of DC power is required to supply an autonomous wireless sensors for satellite health application. Typically such a wireless sensor is composed of: a sensor (DC power comsumption in the range of few $\mu \mathrm{W}$ ), a DC-to-DC convertor, a power management unit and a low-power wireless tranceiver (DC power consumption in the range of the $\mathrm{mW}$ ). However the electromagnetic energy illuminating the antenna panels is almost constant and the DC circuitry (including the power management unit) should be minimal [1]. As represented in Fig. 8 the rectenna can provide a DC power of $1 \mathrm{~mW}$ or higer when it is illuminated by an electric field $\mathrm{E}>38 \mathrm{~V} / \mathrm{m}$. The RFto-DC conversion efficiency of 4CDAA rectenna is computed by using eq. (2) and it is represented in Fig. 9 as function of the E-field illuminating the rectenna under test.

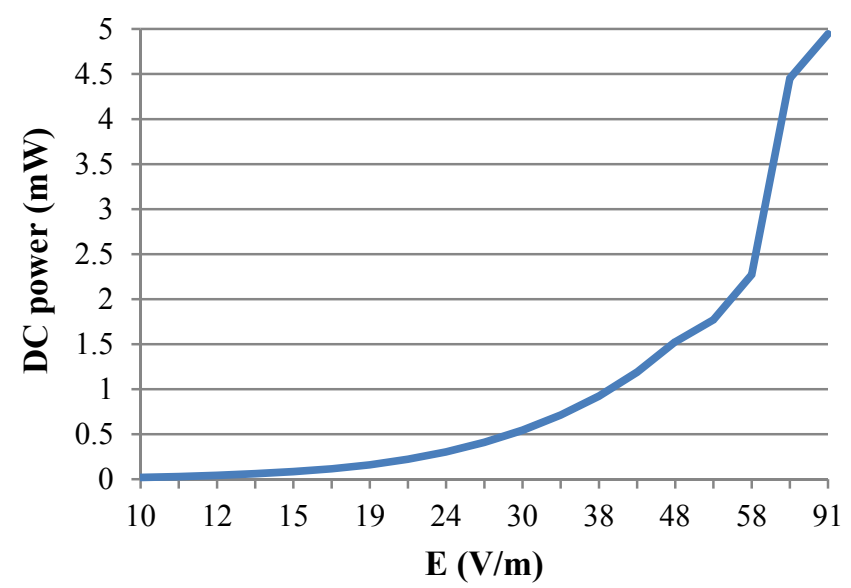

Fig. 8. Measured DC power at the input of a load $(R L=300 \Omega)$ as function of the illuminating E-field

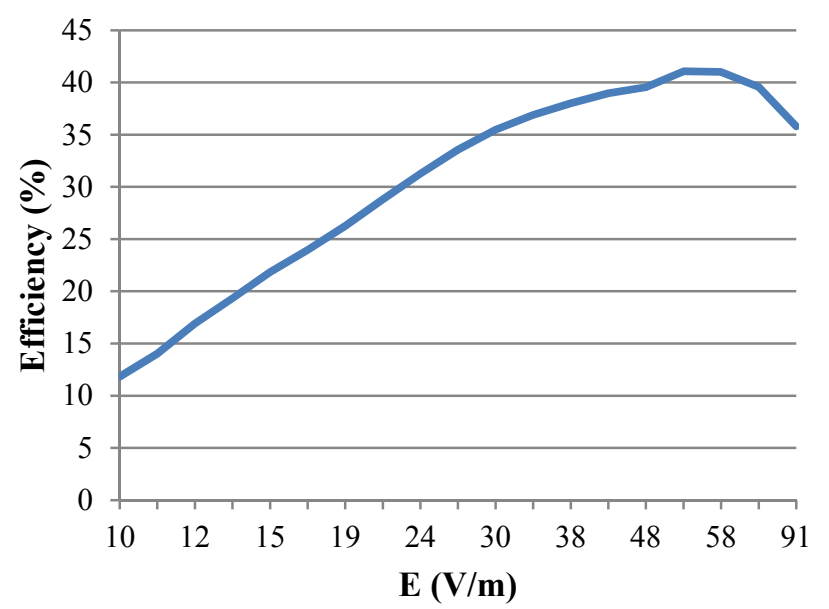

Fig. 9. Rectenna efficiency at the frequency of $12 \mathrm{GHz}$ as function of illuminating E-field for an resistive load of $300 \Omega$.

The reported efficiency is limited by: (i) the ohmic resistance of the selected low-cost silicon diode (ii) by the adopted non-resonant matching technique (iii) the lack of accurate simulation models (in the $\mathrm{Ku}$ band) of the selected commercial diode. The overall performances of the 4CDAA rectenna can be further improved by optimizing the $\mathrm{PCB}$ layout on the bottom side. It was noticed that this botom side layout have a major impact on the impedance matching between the rectifier and the antenna located on the top side of the PCB. Works are under run and more results will be presented at the conference. An efficiency greater than $25 \%$ can be obtained by using a low cost silicon Schottky diode (SMS7630) when the E-field exceeds $19 \mathrm{~V} / \mathrm{m}$. The rectenna is very compact. Its size is $2.5 \mathrm{~cm} \times 2.5 \mathrm{~cm}$ that is square wavelength at $12 \mathrm{GHz}$.

\section{CONCLUSION}

A compact rectenna with an overall size close to square wavelength at $12 \mathrm{GHz}$ was designed and characterized. The proposed design combines an original four cross dipoles antenna array with a rectifier using a single low cost silicon Schottky diode. The experimental results demonstrate that a $\mathrm{DC}$ power greater than $1 \mathrm{~mW}$ can be harvested in the $\mathrm{Ku}$ band $(12 \mathrm{GHz})$ for electric field greater than $38 \mathrm{~V} / \mathrm{m}$. This harvested DC power can be used to power a wireless sensor for satellite health monitoring application. A maximum RF-to-DC conversion efficiency of $41 \%$ was obtained for a load of $300 \Omega$ and an incident electric field of $58 \mathrm{~V} / \mathrm{m}$.

\section{ACKNOWLEDGEMENT}

The authors acknowledge the support of French Space Agency within the framework of several past R\&T contracts.

\section{REFERENCES}

[1] A. Takacs, H. Aubert, S. Fredon, L. Despoisse, H. Blondeaux, "Microwave power harvesting for satellite health monitoring," IEEE Trans. on Microwave Theory Tech, Vol.: 62, Issue: 4 , pp. 1090 - 1098, April 2014.

[2] http://www.skyworksinc.com/Product/511/SMS7630_Series

[3] H. Sun, Y.-X. Guo, M. He, Z. Zhong, "Design of a High-Efficiency 2.45-GHz Rectenna for Low-Input-Power Energy Harvesting", IEEE Antennas Wireless Propag. Lett., Vol. 11, pp. 929-932, 2012.

[4] A. Takacs, H. Aubert, A. Luca, S. Charlot, S. Fredon, L. Despoisse, "Rectenna Design for K Band Application", 2014 European Microwave Conference (EUMC'2014), Rome, Italy, 5-10 Oct'2014.

[5] http://www.skyworksinc.com/uploads/documents/Surface_Mount_Schot tky_Diodes_200041AB.pdf

[6] Skyworks application notes 'Mixer and detector diodes' : http://www.skyworksinc.com/uploads/documents/200826A.pdf

[7] Z. Popovic; E.A. Falkenstein, D. Costinett, R. Zane, "Low-Power FarField Wireless Powering for Wireless Sensors", Proceedings of the IEEE, Vol. 101, No. 6, pp.1397 -1407, June 2013. 